\title{
On the feasibility of a negative polarity electric sail
}

\author{
P. Janhunen \\ Finnish Meteorological Institute, Finland \\ Received: 26 September 2008 - Revised: 2 March 2009 - Accepted: 6 March 2009 - Published: 1 April 2009
}

\begin{abstract}
An electric solar wind sail is a recently introduced propellantless space propulsion method whose technical development has also started. In its original version, the electric sail consists of a set of long, thin, centrifugally stretched and conducting tethers which are charged positively and kept in a high positive potential of $20 \mathrm{kV}$ by an onboard electron gun. The positively charged tethers deflect solar wind protons, thus tapping momentum from the solar wind stream and producing thrust. Here we consider a variant of the idea with negatively charged tethers. The negative polarity electric sail seems to be more complex to implement than the positive polarity variant since it needs an ion gun instead of an electron gun as well as a more complex tether structure to keep the electron field emission current in check with the tether surface. However, since this first study of the negative polarity electric sail does not reveal any fundamental issues, more detailed studies would be warranted.
\end{abstract}

Keywords. Interplanetary physics (Instruments and techniques) - Magnetospheric physics (Storms and substorms) - General or miscellaneous (New fields (not classifiable under other headings))

\section{Introduction}

The electric sail (Janhunen, 2004; Janhunen and Sandroos, 2007) is a recently discovered, completely novel type of space propulsion system concept which uses the solar wind dynamic pressure for producing spacecraft thrust. The technical development of the spinning electric sail (Janhunen, 2006) commenced in 2006 , currently already a long way towards realisation. The spinning electric sail uses the centrifugal force to deploy and stretch out a number of thin, long conducting tethers from the spacecraft (Fig. 1). The tethers are

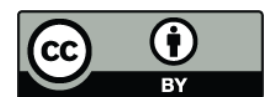

Correspondence to: P. Janhunen (pekka.janhunen@fmi.fi) then charged positively by an onboard electron gun so that their static electric field perturbs the trajectories of the incident solar wind protons, resulting in a momentum transfer from the solar wind plasma stream to the tethers. The force law of the electric sail (Janhunen and Sandroos, 2007) has been used to calculate successful and efficient mission trajectories in the solar system for realistic payloads and other spacecraft characteristics (Mengali et al., 2008a).

The electric sail proposes to use the solar wind dynamic pressure which is $\sim 5000$ times weaker (about $2 \mathrm{nPa}$ at $1 \mathrm{AU}$ ) than the solar radiation pressure used by solar photon sails. The electric sail is, however, able to overcome this apparent shortcoming because the "sail" that intercepts the plasma flow is not a physical obstacle, but a virtual structure made by the electrostatic fields of the charged tethers. The virtual electric sail that forms around each charged tether is typically tens or hundreds of metres in radius which is millions of times larger than the physical width of the tether wires (some tens of micrometres). It is mainly for this reason that the electric sail can be clearly more efficient (i.e. have a larger thrust per propulsion system mass) than a solar photon sail.

In this paper, we consider another variant of the electric sail where the tethers are charged negatively. Like positively charged tethers, also negatively charged bodies are able to perturb the trajectories of solar wind ions, although the details of the interaction differ from the positive case, as we will see below.

The structure of the paper is as follows: Firstly, after briefly reviewing the original positive polarity electric sail concept, we go on to present a semiquantitative analytic theory for the plasma physics of a negatively charged tether placed in solar wind. Secondly, we proceed to estimate the three main current components (ion current, photoelectron current and electron field emission current) that a negatively charged tether gathers from the solar wind. The current is important to evaluate because it determines how much electric power it takes to maintain the tethers at the wanted negative potential. After that, follows a brief survey of some technical

Published by Copernicus Publications on behalf of the European Geosciences Union. 


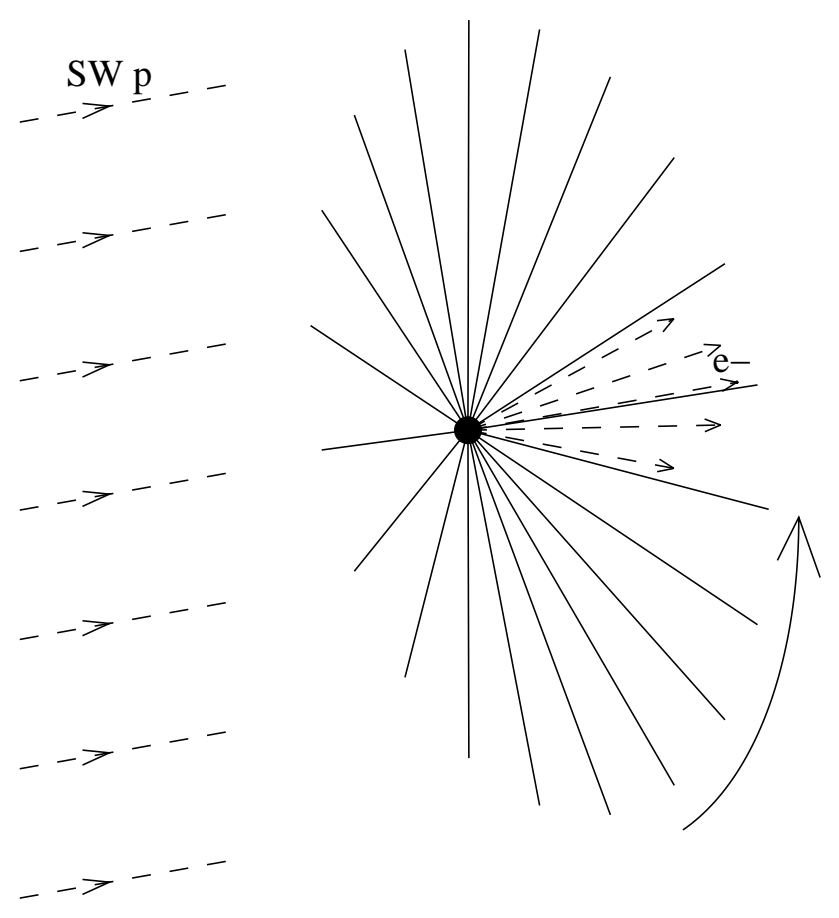

Fig. 1. Schematic description of the original spinning, positive polarity electric sail. Charged, centrifugally stretched tethers gather momentum from the solar wind. The charging is maintained by an electron gun mounted on the spacecraft (middle).

ways for implementing the tether. The paper ends with discussion and conclusions. Plasma simulations of the negative polarity electric sail are outside the scope of this paper, as are quantitative comparisons between the positive and negative polarity variants of the electric sail.

\section{Positive polarity electric sail}

We begin by reviewing the operating principles of the originally proposed positive polarity electric sail (Janhunen, 2004, 2008; Janhunen and Sandroos, 2007). Consider a long, positively charged wire or tether placed in unmagnetised solar wind. We take the solar wind to flow perpendicular to the wire. This is not a restrictive assumption, because any parallel flow component produces no effect since the ion equation of motion in the parallel direction is trivial and not coupled to the rest of the dynamics.

The positively charged wire creates an electron sheath around itself where the potential of the cylindrical wire is approximately

$V(r)=V_{w} \frac{\ln \left[1+\left(r_{0} / r\right)^{2}\right]}{\ln \left[1+\left(r_{0} / r_{w}^{*}\right)^{2}\right]}$

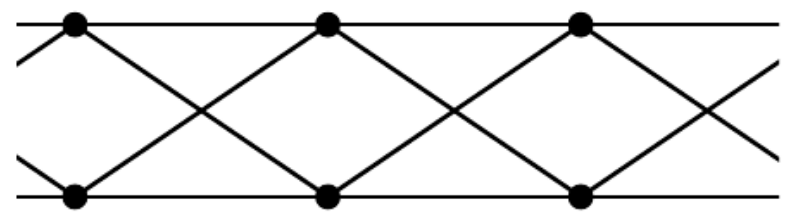

Fig. 2. A four-wire Hoytether. Wire bonding sites are shown by dots.

where

$r_{0}=2 \lambda_{D e}=2 \sqrt{\frac{\epsilon_{\mathrm{o}} T_{e}}{n_{\mathrm{o}} e^{2}}}$.

Here $\lambda_{D e}$ is the electron Debye length, $T_{e}$ is the solar wind electron temperature (on average $T_{e}=12 \mathrm{eV}$ at $1 \mathrm{AU}$ ), $n_{\mathrm{o}}$ is the undisturbed solar wind electron density $\left(n_{\mathrm{o}}=7.3 \mathrm{~cm}^{-3}\right.$ on average at $1 \mathrm{AU}$ ) and $r_{w}^{*}$ is the effective electric radius of the wire, typically $r_{w}^{*} \approx 1 \mathrm{~mm}$. The effective electric radius (Janhunen and Sandroos, 2007) of the tether is larger than the physical radius of the wires $r_{w} \approx 20 \mu \mathrm{m}$ of which the micrometeoroid-resistant multiline tether (Hoyt and Forward, 2001) is constructed (Fig. 2). The potential of the tether relative to the surrounding plasma is $V_{w}$.

The mechanical lifetime of a multiline tether in space depends on its geometry and on the number of wires; four wires typically providing enough long lifetime for the electric sail case according to our numerical estimations based on the micrometeoroid flux model of Grün et al. (1985) ( 1\% breaking probability per $2000 \mathrm{~km}$ of tether during 5 years). The multiline tether can break by two main mechanisms, firstly by numerous small impacts (impactors of $\sim 1 / 3$ of wire width or larger) which break single wires and secondly by one big, centimetre-size impactor which cuts the whole tether at one blow. Clearly, a wide tether is more resistant towards large impactors while a narrow tether handles small impactors better. The optimal tether width in the four-line case is, according to our estimations $\sim 2-3 \mathrm{~cm}$ at $1 \mathrm{AU}$. The optimal width is somewhat wider for asteroid belt missions where the micrometeoroid flux consists of larger particles and narrower for near-Sun missions where the average grain size is smaller (Jehn, 2000). It is also beneficial if the multiline tether is designed to assume a somewhat three-dimensional (i.e. not completely planar) shape in space after deployment, since a completely planar tether could be broken completely by a single small impactor arriving exactly in the tether plane. Different types of multiline tethers and their lifetimes are discussed by Hoyt and Forward (2001).

The solar wind ions experience the potential (Eq. 1) and are deflected in their motion. In the frame of reference of the wire, the total energy of the ion is conserved. Thus, the particle has the same speed after exiting the interaction region than it had originally. Because the direction of the velocity of the ion changes, the particle loses some of its momentum $x$-component, where $x$ is the coordinate along the solar 
wind direction. This lost particle momentum is the reason for the thrust that the tether experiences. The momentum is transferred to the tether by an electric field due to piling up of ions on the sunward side of the tether (i.e., a positive charge cloud) and a corresponding ion depletion region (negative charge cloud) on the antisunward side. Thrust is obtained because the electric field formed between the charge clouds (typically $\sim 1 \mathrm{~V} / \mathrm{m}$ in magnitude) pushes the positively charged tether in the antisunward direction.

According to Janhunen and Sandroos (2007), the force per unit length of the tether is given by

$$
\frac{d F}{d z}=\frac{K m_{p} n_{\mathrm{o}} v^{2} r_{0}}{\sqrt{\exp \left[\frac{m_{p} v^{2}}{e V_{w}} \ln \left(r_{0} / r_{w}^{*}\right)\right]-1}}
$$

where $v$ is the solar wind speed (typically $400 \mathrm{~km} / \mathrm{s}$ ). Equation (3) is derived by finding at which $r$ the potential energy $e V(r)$ of Eq. (1) is equal to the kinetic energy $(1 / 2) m_{p} v^{2}$ of incoming protons and multiplying this distance (the ion deflection distance) by the dynamic pressure of the solar wind and by $K$. Here $K$ is a dimensionless calibration coefficient whose likely value is between 2 and 3 . A test particle calculation of the momentum loss of solar wind protons in potential $V(r)$ of Eq. 1) gives the value $K=3.09$ (Janhunen and Sandroos, 2007). Plasma particle-in-cell (PIC) simulations gives a result which is consistent with $K \approx 3$. However, Janhunen and Sandroos (2007) also showed that an analysis of the PIC result for different values for the electron temperature $T_{e}$ gives a result which is not quite consistent with the functional form of Eq. (3). One can explain away this inconsistency by postulating that due to numerical noise the effective value of the electron temperature in the PIC simulation electron sheath is higher than in the solar wind. If one assumes that this postulate is true, then the PIC simulation results are more consistent with $K \approx 2$ than with $K \approx 3$. Ultimately, only experiments made in space or in the laboratory can give a certainty of the value of $K$. Typical numerical values at $1 \mathrm{AU}$ are $V_{w}=20 \mathrm{kV}, r_{0}=20 \mathrm{~m}$ and $d F / d z=50-100 \mathrm{nN} / \mathrm{m}$ as predicted by Eq. (3).

We assumed above that the solar wind is unmagnetised. This is a good assumption because the ratio of the electron Larmor radius to the electron Debye length is

$$
\frac{r_{L e}}{\lambda_{D e}}=\sqrt{\frac{m_{e}^{2} v_{e}^{2} n_{\mathrm{o}}}{\epsilon_{\mathrm{o}} T_{e} B^{2}}}=\sqrt{\frac{m_{e}}{m_{i}}}\left(\frac{c}{v_{A}}\right) .
$$

At $1 \mathrm{AU}$, typically $v_{A} \approx 80 \mathrm{~km} / \mathrm{s}$ (corresponding to $B=10 \mathrm{nT}$ and $n_{\mathrm{o}}=7.3 \mathrm{~cm}^{-3}$ ) while $\sqrt{m_{e} / m_{i}}=0.023$. Thus at $1 \mathrm{AU}$, $r_{L e} / \lambda_{D e} \approx 90 \gg 1$. When moving radially outward from the Sun, this ratio does not change since it is proportional to the Alfvén speed $v_{A}$. The Alfvén speed does not vary because $\rho \sim 1 / r^{2}$ and $B \sim 1 / r$ in the equatorial plane. Thus the magnetic field can be neglected when considering electron motion in the sheath region. For ions, the Larmor radius is still larger by factor $\approx 70$ so they can be assumed to be unmagnetised as well.

The induced electromotive force, due to the solar wind magnetic field, is generally insignificant compared to the tether voltage. For example, for a typical $10 \mathrm{nT}$ field, $400 \mathrm{~km} / \mathrm{s}$ solar wind speed and $20 \mathrm{~km}$ long tethers, the maximum induced potential along the tether is only $80 \mathrm{~V}$. For missions going near the Sun, e.g. at Mercury distance, the field can sometimes be as large as $60 \mathrm{nT}$, which would give a $0.5 \mathrm{kV}$ potential difference. Since typical voltages are $\sim 20 \mathrm{kV}$ for the positive polarity sail, one can conclude that the induced voltage by the magnetic field is not significant and, in most cases, can be ignored when analysing electric sails.

The outward surface electric field on the tether wires is typically $100-200 \mathrm{MV} / \mathrm{m}$. This field is still not high enough for it to cause a significant emission of ions or ion clusters from the surface. It is conceivable that some local microscopic protrusions (e.g. whiskers) may be torn away by the electrostatic force. Such protrusions might exist on the metal surface originally or be caused by micrometeoroid cratering in space. If this happens, it should not cause any problems, since the expelled positively charged particles and fragments exit permanently to space. Only an electric field, which is in the range $5-10 \mathrm{GV} / \mathrm{m}$ or even higher, could be able to extract larger amounts of ions from a metal surface without the field being amplified by local protrusions.

Equation (3) is formulated to agree with one- and twodimensional self-consistent plasma simulations of Janhunen and Sandroos (2007). The plasma simulations are not stationary, but include explicitly the ramp-up phase of the tether voltage. During the ramp-up phase, a certain fraction of ambient electrons get trapped in the potential well. Consequently, Eq. (3) includes the extra shielding effect of these trapped electrons. As shown by Sanmartín et al. (2008), if trapped electrons were absent, the potential structure would be wider. Presumably, the thrust would also then be larger than predicted by Eq. (3). After the present paper was submitted, arguments were found supporting the view that trapped electrons actually tend to be removed by orbit chaotisation in a realistic electric sail geometry and that the positive polarity electric sail thrust could be roughly five times larger than predicted by Eq. (3) (Janhunen, 2009). In the present paper, performance comparisons of the positive and negative sails are not attempted and from now on we concentrate on analysing the negative sail variant as a standalone concept.

\section{Negative polarity electric sail}

A negatively charged tether attracts solar wind ions and repels electrons so that an ion sheath gets formed around the tether. Electrons (temperature $\sim 10 \mathrm{eV}$ ) are cold compared to the solar wind ion bulk flow kinetic energy $(\sim 1 \mathrm{keV})$ and to the tether potential $(>1 \mathrm{kV})$. They are thus pushed out to 
a distance where the tether potential is almost completely masked by ions. Consequently, there is an electron hole inside which only ions exist. Outside the electron hole, the plasma properties do not differ markedly from the ambient solar wind plasma.

In the positive polarity electric sail, some electrons get trapped by the potential structure when the voltage is turned on (Janhunen, 2004). A particle may get trapped by a potential structure only if the potential structure deepens by more than the particle's original kinetic energy during the time the particle resides in the structure. Thus, only those particles that initially moved very slowly with respect to the tether can be trapped. The number of trapped particles does not depend on the deepening rate of the potential structure. A faster deepening rate implies a shorter duration of the process so that fewer particles occur on the structure, but on the other hand the width of the energy window from which particles are trapped increases. These two effects cancel each other out so that the number of trapped particles does not depend on how rapidly the potential of the tether is turned on.

The solar wind electrons are approximately isotropic in the sense that their bulk velocity $(\sim 400 \mathrm{~km} / \mathrm{s})$ is less than their random thermal speed $(\sim 1600 \mathrm{~km} / \mathrm{s})$. Thus, the distribution contains a fair fraction of electrons with velocities close to zero. The situation is completely different for solar wind ions, because their thermal spread is only $\sim 10 \%$ of the bulk speed. Thus, extremely slow ions (relative to the spacecraft) that could become trapped are very few in the source distribution. Consequently, no trapped ions are expected to exist in the potential structure of a negatively charged tether placed in the solar wind.

Consider an arbitrary two-dimensional negative potential well $V(x, y) \leq 0$. Our task is now to estimate the ion density inside the potential in the absence of trapped ions. For simplicity, we assume that there is no preferred direction in ion velocity space, i.e. that the tether is approached by ion streams from all perpendicular directions isotropically. This assumption is not realistic because the solar wind ions arrive only from one direction, but we shall make it here in order to obtain an analytically tractable problem which hopefully gives some rough guidelines to the real situation. The ion distribution far from the tether is then the ring distribution,

$f_{\mathrm{o}}\left(v^{\prime}\right)=\frac{n_{\mathrm{o}}}{2 \pi v_{\mathrm{o}}} \delta\left(v^{\prime}-v_{\mathrm{o}}\right)$

where $v_{\mathrm{o}}$ is the ion stream speed, taken to be equal to the solar wind bulk velocity, $n_{\mathrm{o}}$ is the ambient ion density and $\delta$ is the Dirac delta function. By Liouville's theorem, which is valid for collisonless plasma, the distribution function at point $(x, y)$ inside the potential structure is given by

$f\left(x, y, v_{x}, v_{y}\right)=f_{\mathrm{o}}\left(v^{\prime}\right) \chi\left(x, y, v_{x}, v_{y}\right)$

where energy conservation fixes the original speed of the particle to be

$v^{\prime}=\sqrt{v_{x}^{2}+v_{y}^{2}+2 e V(x, y) / m_{i}}$ and where the accessibility characteristic function $\chi\left(x, y, v_{x}, v_{y}\right)$ is equal to one if backward trajectory integration from $\left(x, y, v_{x}, v_{y}\right)$ reaches a point which is outside the potential structure and zero otherwise. From Eqs. (5-7) we obtain

$$
\begin{aligned}
f\left(x, y, v_{x}, v_{y}\right)= & \frac{n_{\mathrm{o}}}{2 \pi v_{\mathrm{o}}} \delta\left[\sqrt{v_{x}^{2}+v_{y}^{2}+\frac{2 e}{m_{i}} V(x, y)}-v_{\mathrm{o}}\right] \\
& \chi\left(x, y, v_{x}, v_{y}\right) .
\end{aligned}
$$

Equation (8) can be integrated to give the ion density inside the potential structure

$$
\begin{aligned}
n(x, y) & =\int d v_{x} d v_{y} f\left(x, y, v_{x}, v_{y}\right) \\
& \leq \frac{n_{\mathrm{o}}}{v_{\mathrm{o}}} \int_{0}^{\infty} d v v \delta\left[\sqrt{v^{2}+2 e V(x, y) / m_{i}}-v_{\mathrm{o}}\right] \\
& =\frac{n_{\mathrm{o}}}{v_{\mathrm{o}}} \int_{-v_{\mathrm{o}}}^{\infty} d u\left(u+v_{\mathrm{o}}\right) \delta(u) \\
& =n_{\mathrm{o}} .
\end{aligned}
$$

Here we made a change of variable

$u=\sqrt{v^{2}+2 e V(x, y) / m_{i}}-v_{\mathrm{o}}$

so that

$d u=\frac{v d v}{\sqrt{v^{2}+2 e V(x, y) / m_{i}}}$

and made use of the fact that $\chi\left(x, y, v_{x}, v_{y}\right) \leq 1$. Thus we obtain an important and simple result that the ion density inside the potential structure is at most equal to the ambient density $n_{\mathrm{o}}$, irrespective of the functional form of the potential structure $V(x, y)$. The result holds well for any two-dimensional potential (Laframboise and Parker, 1973).

Knowing now that the ion density is everywhere limited by $n_{\mathrm{o}}$ and the electron density is approximately zero inside some radius $R$ and approximately equal to $n_{\mathrm{o}}$ outside $R$ (the transition is sharp since the electrons are cold), we can calculate the functional form of the potential $V(x, y)=V(r)$ under the assumption of maximum ion shielding, defined by $\chi\left(x, y, v_{x}, v_{y}\right)=1$ everywhere so that the inequality in Eq. (11) becomes an equality. At radius $R$, it holds that the ions completely shield the negatively charged tether inside, i.e.

$-e \pi R^{2} n_{\mathrm{o}}=\lambda=2 \pi \epsilon_{\mathrm{o}} r_{w} E_{0}$

where $\lambda<0$ is the line charge, $r_{w}$ the radius and $E_{0}<0$ the surface electric field of the tether. Solving Eq. (12) for $R$ we obtain

$R=\sqrt{\frac{2 \epsilon_{\mathrm{o}} r_{w}\left(-E_{0}\right)}{e n_{\mathrm{o}}}}$. 
The electric field at a radius $r, r_{w}<r<R$ is given by Gauß' law,

$$
\begin{aligned}
E(r) & =\frac{\left(\lambda+e \pi n_{\mathrm{o}} r^{2}\right)}{2 \pi \epsilon_{\mathrm{o}} r} \\
& =\left(\frac{r_{w}}{r}\right) E_{0}+\frac{e n_{\mathrm{o}} r}{2 \epsilon_{\mathrm{o}}} .
\end{aligned}
$$

Integrating Eq. (14) we obtain the potential

$$
\begin{aligned}
V(r) & =\int_{r}^{R} d r E(r) \\
& =\left(r_{w} E_{0}\right)\left[\ln \left(\frac{R}{r}\right)-\frac{1}{2}\right]-\frac{T_{e}}{4 e}\left(\frac{r}{\lambda_{D e}}\right)^{2}
\end{aligned}
$$

where we have expressed the result in terms of the electron Debye length $\lambda_{D e}=\sqrt{\epsilon_{\mathrm{o}} T_{e} /\left(n_{\mathrm{o}} e^{2}\right)}$.

Equation (15) defines a solution for the potential valid for cold electrons, no trapped ions and the entire phase space accessible for traversing ions. If the entire phase space is not accessible for traversing ions, then $\chi\left(x, y, v_{x}, v_{y}\right)$ vanishes at some points. In this case the ion density may become smaller than what was assumed in the above calculation, and the non-logarithmic terms in Eq. (15) should be damped by some factor which is less than unity. In what follows, we shall not consider this correction, because the most important term in Eq. (15) is the logarithmic one. Including this correction would tend to decrease ion shielding and therefore to increase the propulsive force, so our assumption $\chi\left(x, y, v_{x}, v_{y}\right)=1$ gives a lower limit for the propulsive force.

In reality the solar wind arrives from only one direction, so the solution is no longer symmetrical. In any case, to roughly estimate the force acting on the tether, we must estimate the extent of the potential well which is able to cause a large-angle turning of incoming ions. This amounts to finding the radial distance (ion deflection distance) $r_{s}$ such that $V\left(r_{s}\right)=V_{i} \equiv-(1 / 2) m_{i} v_{\mathrm{o}}^{2} / e$. The solution for $r_{s}$ can be expressed in terms of Lambert's $W$-function, also called the product $\log$ function. For any $x, W(x)$ is defined to be equal to a solution $y$ of the equation

$x=y e^{y}$.

The solution for the ion deflection distance is

$r_{s}=R \sqrt{-W\left[-\exp \left(-2 \frac{V_{i}}{r_{w} E_{0}}-1\right)\right]}$

and a rough estimate for the thrust per unit length $d F / d z$ is the solar wind dynamic pressure $P_{\mathrm{dyn}}=m_{i} n_{\mathrm{o}} v_{\mathrm{o}}^{2}$ multiplied by the extent of the strong ion scattering region $2 r_{s}$,

$\frac{d F}{d z} \approx m_{i} v_{\mathrm{o}}^{2} \sqrt{\frac{8 \epsilon_{\mathrm{o}}}{e} n_{\mathrm{o}} V_{0} W\left[-\exp \left(-\frac{m_{i} v_{\mathrm{o}}^{2}}{e\left|V_{0}\right|}-1\right)\right]}$

where the potential parameter $V_{0}=r_{w} E_{0}$. For $V_{0}$ not much larger than $V_{i}$, Lambert's $W$-function in Eq. (18) is evaluated at a small negative argument for which it is enough to take the first term in $W$ 's Taylor series

$W(x)=x-x^{2}+(3 / 2) x^{3}+O(x)^{4}$.

Thus, with $W(x) \approx x$, Eq. (18) becomes

$$
\begin{aligned}
\frac{d F}{d z} & \approx m_{i} v_{\mathrm{o}}^{2} \sqrt{\frac{8 \epsilon_{\mathrm{o}}}{e} n_{\mathrm{o}}\left|V_{0}\right|} \exp \left(-\frac{m_{i} v_{\mathrm{o}}^{2}}{2 e\left|V_{0}\right|}-\frac{1}{2}\right) \\
& =1.72 P_{\mathrm{dyn}} \sqrt{\frac{\epsilon_{\mathrm{o}}\left|V_{0}\right|}{n_{\mathrm{o}} e}} \exp \left(-\frac{(1 / 2) m_{i} v_{\mathrm{o}}^{2}}{e\left|V_{0}\right|}\right) .
\end{aligned}
$$

where we used $\sqrt{8} e^{-1 / 2}=1.72$. Recall that the solar wind dynamic pressure is $P_{\mathrm{dyn}}=m_{i} n_{\mathrm{o}} v_{\mathrm{o}}^{2}$.

The potential of the tether, with respect to the surrounding plasma, is obtained from Eq. (15) by setting $r=r_{w}$. In this case, the last term which is proportional to $T_{e}$ is vanishingly small and we obtain

$V_{w}=V\left(r_{w}\right)=V_{0}\left[\ln \left(\frac{R}{r_{w}}\right)-\frac{1}{2}\right]$

Equation (20), or alternatively the more accurate version Eq. (18), is the main result of this section. It tells us that the effective electric sail width of a negatively charged tether contains, as the essential factor, an "effective Debye length"

$\lambda_{D}^{\mathrm{eff}}=\sqrt{\frac{\epsilon_{\mathrm{o}}\left|V_{0}\right|}{n_{\mathrm{o}} e}}$

where instead of the electron temperature the potential parameter $V_{0}=r_{w} E_{0}$ appears (recall that $E_{0}$ is the wire surface electric field). This length $\lambda_{D}^{\text {eff }}$ is potentially large, leading to a potentially large force per tether length. For example, in average solar wind at $1 \mathrm{AU}$ and with $V_{0}=1 \mathrm{kV}, \lambda_{D}^{\text {eff }}=87 \mathrm{~m}$.

However, before being able to realise the potential thrust benefit of the negative polarity electric sail, several questions need to be addressed: How large a current does a negatively charged tether gather from the solar wind plasma? How large a surface electric field $E_{\mathrm{o}}$ can be safely maintained without a risk of an electric breakdown of some sort? Is the ion gun, which is necessary to maintain the negative potential, difficult to construct? Does one need significant amounts of propellant to create the ions? We will now consider these questions.

To control the motion of the tethers, the tether potentials must be known and controllable. This can be accomplished by monitoring the spacecraft potential with respect to the surrounding plasma by using a particle detector (ion detector in negative polarity sail, electron detector in positive polarity sail). The tether potentials are controlled by potentiometers (tunable resistors) that are installed between each tether and the spacecraft. For each tether, its potential is the sum of the spacecraft potential and the potentiometer voltage. The potentiometer voltage can be measured electrically and it can be controllably changed by changing the resistance of the 
potentiometer. The turning of the sail is possible using an algorithm resembling helicopter flight control where the tether potentiometer settings are modifying cyclically and in sync with the rotation when one wants to tilt the sail (Janhunen, 2006).

\section{Current gathered by negatively charged tether}

Consider a negatively charged tether immersed in solar wind plasma. Our task is to estimate how much current per unit length the tether gathers from the plasma if it is kept at potential $V_{w}$.

We consider three sources of gathered current: the thermal ion current arriving from the surrounding plasma, current carried by solar ultraviolet (UV) radiation induced photoemission of electrons from the tether surface and finally current due to field emission of electrons (Fowler-Nordheim current) at the tether surface.

\subsection{Thermal ion current}

Because the solar wind is very tenuous, the plasma is collisionless to a very good approximation. The tether acts as a cylindrical probe immersed in plasma and the ion current can be calculated by the orbital motion limited (OML) theory (Allen, 1992). The current per unit length is

$$
\left(\frac{d I}{d z}\right)_{\text {th. }}=e n_{\mathrm{o}} \sqrt{\frac{e V_{w}}{m_{p}}} 2 r_{w} .
$$

For a self-contained derivation of this expression, see Appendix B of Janhunen and Sandroos (2007). This current is $\sqrt{m_{e} / m_{p}} \approx 43$ times smaller than the corresponding electron current in the positive electric sail case and can nearly always be neglected.

\subsection{Photoelectron current}

A sunlit object, such as a conducting tether, emits photoelectrons due to solar UV radiation. If the tether is positively charged, the photoelectrons cause no macroscopic current since they are rapidly attracted back to the tether. When the tether is negatively charged, however, photoelectrons are repelled by the tether and therefore they contribute directly to the current gathered by the tether. Notice that the photoelectron current is independent of the tether voltage, but depends only on the tether surface properties, the solar UV spectrum and the solar distance.

Grard (1973) estimated the photoelectron emission at $1 \mathrm{AU}$ from selected materials as follows: aluminium oxide $42 \mu \mathrm{A} \mathrm{m}^{-2}$, gold $29 \mu \mathrm{A} \mathrm{m}^{-2}$, stainless steel $20 \mu \mathrm{A} \mathrm{m}^{-2}$, graphite $4 \mu \mathrm{A} \mathrm{m}^{-2}$. The spacecraft photoelectron yields inferred from orbital data are typically somewhat higher than those based on laboratory measurements of Grard (1973). For example, Nakagawa et al. (2000) determined the photoelectron current for the GEOTAIL spacecraft to be $85 \pm 33 \mu \mathrm{A} \mathrm{m}^{-2}$. Our present baseline material for constructing electric sail tethers is aluminium whose surface has the natural oxidation layer formed by atmospheric oxygen. We shall here adopt Grard's aluminium oxide value as representative for the tethers so that we have

$$
\left(\frac{d I}{d z}\right)_{\text {photoel. }}=42 \mu \mathrm{Am}^{-2} \times 2 r_{w}\left(\frac{1 \mathrm{AU}}{r}\right)^{2} \text {. }
$$

With average solar wind density at $1 \mathrm{AU}$ and with $V_{w}=20 \mathrm{kV}$, the photoelectron current is $43 \%$ of the electron current of the corresponding positive electric sail case.

Both the thermal plasma current and the photoelectron current scale as $1 / r^{2}$ where $r$ is the distance from the Sun. Thus both the ion thermal current and the photoelectron current are conveniently modest in magnitude. They do not appear to present a problem for the realisation of a negative polarity electric sail.

\subsection{Electron field emission current}

A negatively charged electrode may emit electrons at a much lower surface field than a positively charged electrode can emit ions. Electron emission from sharp, well-cleaned tips follows closely to the Fowler-Nordheim field emission law and its later modifications taking into account finite temperature and other refinements (Alpert et al., 1964; Noer, 1982; Halbritter, 1983) and takes place typically at $1-2 \mathrm{GV} / \mathrm{m}$ surface field. (Since the dependence of the current density on the electric field $E$ is proportional to $E^{2} \exp (-1 / E)$, the emission increases rapidly from insignificant to large values.) For broad-area electrodes containing attached dust, local impurities and adsorbed gases, however, significant electron field emission is typically observed already at $\sim 100$ times smaller fields of order $10 \mathrm{MV} / \mathrm{m}$ (Noer, 1982; Halbritter, 1983).

Basically, all experimental investigations of electron field emission have taken place in ground-based laboratories rather than in space. In the laboratory, there are always two electrodes present, anode and cathode. There are experimental indications that the onset and nature of electron field emission at the cathode also depends on the properties of the anode (Noer, 1982). The physical mechanism is possibly that relatively weak but very localised electron beams initially emitted by the cathode knock loose some ions from the anode upon impacting it. The ions are then accelerated by the electric field back to the cathode and impact it near the original electron emission spot. This may cause further secondary electron emissions at the cathode local emission site. Ions in the space between the electrodes can also be produced by electron impact ionisation of neutral atoms outgassed from the anode by local heating caused by the cathode-emitted electron beams.

These complicated interaction and feedback phenomena between the cathode and anode, while always present in the laboratory, are absent in space where the other electrode is 
formed by the surrounding space plasma. It might, therefore, be the case that a broad-area cathode in space tolerates higher surface fields than in the laboratory. There is in practice no other way to find this out other than to perform an experiment in space with a thin cathode wire.

All materials, including metals, outgas to some extent in a vacuum. Part of the outgassed molecules can become ionised by solar UV radiation while moving out from the tether. If ionisation takes place while the molecule is inside the potential structure of the tether, it may form a trapped ion. We have estimated the magnitude of this effect and found that under some circumstances, such outgassing produced ions might produce unwanted trapped ions to an extent that matters. An easy fix to this potential problem, if it occurs, is to reset the potential periodically to clean out the unwanted trapped ions from the potential structures of the tethers.

\section{Technical design}

\subsection{Ion gun}

The negative polarity sail needs an ion gun instead of an electron gun to keep itself charged. Although an ion gun is a more complicated device than an electron gun, its construction should not present any fundamental problems. Importantly, the number of ions that one must emit over a reasonable operational lifetime of the electric sail (some years), is not so large that the mass of the propellant from which the ions are created would be a significant item in the spacecraft mass budget. The ion gun is effectively equivalent to a very high specific impulse ion engine. Such a device needs mainly power, but does not consume much propellant. Since the aim is to produce current rather than mass flow, light ions would be preferable to heavy ones in this case, in order to minimise propellant mass. Since ion engines typically use xenon (atomic mass 131), we shall consider it also here, however. If the electric sail tethers are charged to $20 \mathrm{kV}$, the speed of the emitted $\mathrm{Xe}^{+}$ions is $170 \mathrm{~km} / \mathrm{s}$. Recent developments of fourgridded ion engines should make such high exhaust speeds reachable (Fearn, 2005). At such a high specific impulse, even a high-powered engine uses relatively little propellant. As noted above, by replacing xenon by a lighter element such as argon, the propellant mass consumption could be lowered further if needed. Overall, then, if and when the power is available to feed it, the ion gun which is needed to keep the negative polarity electric sail charged should not present insurmountable problems.

The extra thrust provided by the ion gun is almost negligible (less than $1 \mathrm{mN}$ even if using xenon) in comparison with the electric sail thrust, because the ion gun specific impulse is very high and its power is only modest.

\subsection{Tubular tether}

Consider a hollow tubular tether with radius $r_{w}$, wall thickness $h\left(h \ll r_{w}\right)$, material mass density $\rho_{m}$ and conductivity $\sigma$. The tether mass per unit length is

$\frac{d m}{d z}=\rho_{m} \pi h\left(2 r_{w}-h\right)$.

If we use surface electric field $E_{0}$, the tether generates force per unit length

$\frac{d F}{d z}=1.72 P_{\mathrm{dyn}} \sqrt{\frac{\epsilon_{\mathrm{o}}\left|V_{0}\right|}{n_{\mathrm{o}} e}} \exp \left(-\frac{(1 / 2) m_{i} v_{\mathrm{o}}^{2}}{e\left|V_{0}\right|}\right)$

where $V_{0}=r_{w} E_{0}$. Assuming that the surface electric field is such that electron field emission current is negligible, other current components except the photoelectron current (Eq. 24) can be neglected. The power consumption per unit length is then the current obtained from Eq. (24) multiplied by the tether voltage (Eq. 21). The result can be written in the form

$\frac{d P}{d z}=\left(42 \mu \mathrm{Am}^{-2}\right) \times 2 r_{w} V_{0}\left[\ln \left(\frac{\lambda_{D}^{\text {eff }}}{r_{w}}\right)-\frac{1-\ln 2}{2}\right]$

where $\lambda_{D}^{\text {eff }}$ is defined by Eq. (22).

As a performance metric, we use the specific acceleration $a_{\text {spec }}$, defined here to be the obtained thrust divided by the mass of the tether as obtained from Eqs. (25) and (26), or $a_{\text {spec }}=(d F / d z) /(d m / d z)$. Figure 3 shows the specific acceleration $a_{\text {spec }}$ and the specific power [consumed power divided by obtained thrust, $(d P / d z) /(d F / d z)]$ as a function of the tether radius of a tubular aluminium tether with $10 \mu \mathrm{m}$ wall thickness in average solar wind at $1 \mathrm{AU}$, for two values of the allowed surface electric field $E_{0}=5$ and $10 \mathrm{MV} / \mathrm{m}$. One sees that the result depends strongly on the value of $E_{0}$ adopted. For $E_{0}=10 \mathrm{MV} / \mathrm{m}$, for example, we obtain at the optimum point a specific acceleration of $8 \mathrm{~mm} / \mathrm{s}^{2}$ at $\sim 20 \mathrm{kV}$ voltage, consuming only $\sim 1.3 \mathrm{~W} / \mathrm{mN}$ of electric power. Assuming that the spacecraft has e.g. 100 tethers of $20 \mathrm{~km}$ length each, we obtain in this case a total thrust of $0.42 \mathrm{~N}$, tether mass $50 \mathrm{~kg}$ and power consumption $530 \mathrm{~W}$. If the power system, tether reels and ion gun would weigh another $50 \mathrm{~kg}$ and the payload e.g. $300 \mathrm{~kg}$ so that the total mass would be $400 \mathrm{~kg}$, the propulsive acceleration would be about $1 \mathrm{~mm} / \mathrm{s}^{2}$. This level of acceleration is already adequate for performing many manoeuvres in the solar system (Mengali et al., 2008a,b).

The tether mass for a tubular tether is directly proportional to the wall thickness which was here assumed to be $10 \mu \mathrm{m}$. For example, with a thinner $5 \mu \mathrm{m}$ wall the specific acceleration would be two times higher and in the above example the tether mass would be $25 \mathrm{~kg}$ instead of $50 \mathrm{~kg}$.

The quantity $a_{\text {spec }}$ is useful because it is simple to calculate and does not depend on other parameters. In a more detailed performance analysis, one should use a more advanced figure 


\section{Tubular tether}

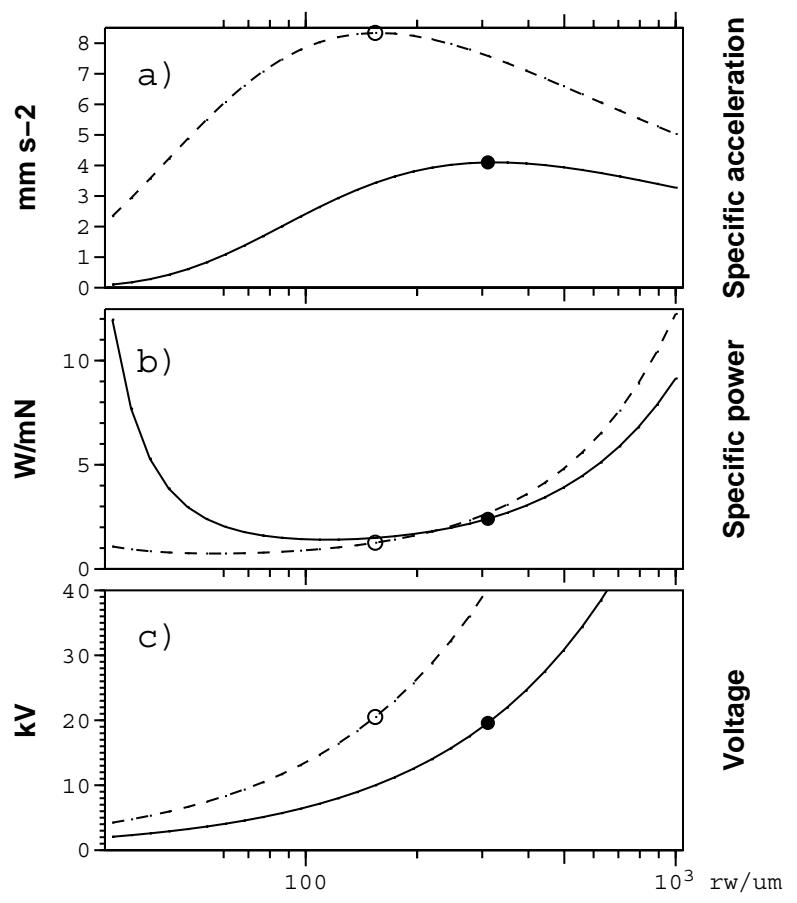

Fig. 3. Specific acceleration (a), specific power consumption (b) and voltage (c) of a tubular, negatively charged aluminium tether with $10 \mu \mathrm{m}$ wall thickness. The logarithmic horizontal axis is the radius $r_{w}$ of the tether in micrometres. Solid curves correspond to allowed surface electric field $E_{0}=5 \mathrm{MV} / \mathrm{m}$ and dashed curves to $E_{0}=10 \mathrm{MV} / \mathrm{m}$. Points of maximum specific acceleration are marked on the curves.

of merit which takes explicitly into account the mass of the spacecraft power system, tether reels and the ion gun. However, for applications where the payload mass is in any case rather large compared to the sail propulsion system mass, the exact mass of the propulsion system is not too significant. In this type of application, it is more important that the electric sail propulsion system can generate enough thrust without having to make the total tether length excessive.

In practice, instead of a tubular tether, one might prefer to use related but somewhat different tethers such as quasitubular "wiresock" tethers constructed by wire-wire bonding from e.g. 10-30 metal wires of $\sim 20 \mu \mathrm{m}$ thickness. However, analysing this and other kinds of tether structures, is outside the scope of this paper.

The most important parameter whose exact value remains unknown is the allowed surface electric field $E_{0}$, i.e., how large surface electric field $E_{0}$ the tether tolerates before field emission from the tether (probably mainly from points damaged by micrometeoroids) becomes comparable to or larger than the photoelectron current. We think it is likely that a value $E_{0}=5 \mathrm{MV} / \mathrm{m}$ should be rather safe, but it is not out of the question that also larger values could be used. Experiments would be needed to ascertain this.

\section{Discussion and conclusions}

The negative polarity sail current has three components: ion current, photoelectron current and electron field emission current. The ion current is small and it scales by the solar wind density $\left(1 / r^{2}\right)$. The photoelectron current is larger (roughly similar to typical current in positive polarity sail) and it also scales as $1 / r^{2}$. The sum of ion and photoelectron currents, therefore, scales as $1 / r^{2}$ and is nearly independent of the tether voltage, because the ion current part is small. Currents that scale as $1 / r^{2}$ are nice because their associated power consumption then scales in the same way $\left(1 / r^{2}\right)$ as the power produced by solar panels. The electron field emission current is independent of $r$, but it depends strongly on the tether voltage. The level of usable surface electric field $E_{0}$, before significant electron field emission sets in at some point on the tether, is the main parameter in the negative electric sail concept whose exact value remains inaccurately known, due to lack of experimental data on long cathode wires without nearby anodes in the ultra-high vacuum of space.

Recently, Choinière and Gilchrist (2007) studied the sheath extent and current collection of negative tethers in moving plasma by self-consistent steady state 2-D Vlasov simulation and under the assumption of no trapped particles. Their calculation method could probably be easily extended to produce more accurate thrust estimates than what we have provided here.

In principle, the thrust of the negative polarity electric sail also receives a contribution from the impulse given by ions that hit the wires. This thrust contribution can be estimated to be negligibly small, however, for typical parameter values, so we do not discuss it explicitly.

If significant electron field emission occurs, it takes place at a few points containing dielectric impurities, micrometeoroid damage or other anomalies along the tether. We might speculate with the possibility of "cleaning" the tether of some of the high field emission sites by running it in high positive polarity mode for some time, hoping that Coulomb repulsion extracts the unwanted material from the surface. This possibility, while speculative, would in any case be accessible to experimental investigation.

In conclusion, the negative polarity electric sail might be a feasible concept to implement in its own right, although it has a set of issues mainly related to electron field emission from negatively charged wires that would need to be addressed. The question of whether the negative polarity variant would provide some performance benefit compared to the original positive polarity electric sail depends mainly on whether trapped electrons exist or not in the positive polarity sail. This question is presently under active study and one should wait for the answer before drawing final conclusions. In any case, the thrust level of both the positive and negative electric sail concepts has the potential to be quite high in comparison to presently existing space propulsion methods. 
Acknowledgements. The author thanks Petri Toivanen and Arto Sandroos for many discussions related to the topic and acknowledges the much larger international electric sail team for background support. The work was supported by the Academy of Finland. The author also wants to express special thanks to both referees for their thorough scrutiny of the paper using a multitude of approaches.

Topical Editor I. A. Daglis thanks two anonymous referees for their help in evaluating this paper.

\section{References}

Allen, J. E.: Probe theory - the orbital motion approach, Physica Scripta, 45, 497-503, 1992.

Alpert, D., Lee, D. A., Lyman, E .M., and Tomaschke, H. E.: Initiation of electrical breakdown in ultrahigh vacuum, J. Vacuum Sci. Tech., 1, 35-50, 1964.

Choinière, È. and Gilchrist, B. E.: Self-consistent 2-D kinetic simulations of high-voltage plasma sheaths surrounding ion-attracting conductive cylinders in flowing plasmas, IEEE Trans. Plasma Sci., 35, 7-22, 2007.

Fearn, D.: The application of gridded ion thrusters to high thrust, high specific impulse nuclear-electric missions, J. British Interplanetary Soc., 58, 257-267, 2005.

Grard, R. J. L.: Properties of the satellite photoelectron sheath derived from photoemission laboratory measurements, J. Geophys. Res., 78, 2885-2906, 1973.

Grün, E., Zook, H. A., Fechtig, H., and Giese, R. H.: Collisional balance of the meteoritic complex, Icarus, 62, 244-272, 1985.

Halbritter, J.: Enhanced electron emission and its reduction by electron and ion impact, IEEE Trans. Electrical Insulation, EI-18, 253-261, 1983.

Hoyt, R. and Forward, R. L.: Alternate interconnection Hoytether failure resistant multiline tether, US Pat. 6286788 B1, 2001.
Laframboise, J. G. and Parker, L. W.: Probe design for orbit-limited current collection, Phys. Fluids, 16(5), 629-636, 1973.

Janhunen, P.: Electric sail for spacecraft propulsion, J. Propulsion Power, 20(4), 763-764, 2004.

Janhunen, P.: Electric sail for producing spacecraft propulsion, US Pat. 11/365875 (pending), 2006.

Janhunen, P. and Sandroos, A.: Simulation study of solar wind push on a charged wire: basis of solar wind electric sail propulsion, Ann. Geophys., 25, 755-767, 2007, http://www.ann-geophys.net/25/755/2007/.

Janhunen, P.: The electric sail - a new porpulsion method which may enable fast missions to the outer solar system, J. British Interplanetary Soc., 61, 322-325, 2008.

Janhunen, P.: Increased Electric Sail thrust through removal of trapped shielding electrons by orbit chaotisation due to spacecraft body, Ann. Geophys., submitted, 2009.

Jehn, R.: An analytical model to predict the particle flux on spacecraft in the solar system, Planet. Space Sci., 48, 1429-1435, 2000.

Mengali, G., Quarta, A., and Janhunen, P.: Electric sail performance analysis, J. Spacecraft Rockets, 45, 122-129, 2008.

Mengali, G., Quarta, A. A., and Janhunen, P.: Considerations of electric sailcraft trajectory design, J. British Interplanetery Soc., 61, 326-329, 2008.

Nakagawa, T., Ishii, T., Tsuruda, K., Hayakawa, H., and Mukai, T.: Net current density of photoelectrons emitted from the surface of the GEOTAIL spacecraft, Earth Planet Space, 52, 283-292, 2000.

Noer, R. J.: Electron field emission from broad-area electrodes, Appl. Phys. A., 28, 1-24, 1982.

Sanmartín, J. R., Choinière, E., Gilchrist, B. E., Ferry, J.-B., and Martínez-Sánchez, M.: Bare-tether sheath and current: comparison of asymptotic theory and kinetic simulations in stationary plasma, IEEE Trans. Plasma Phys., 36, 2851-2858, 2008. 